\title{
Nice work - but is it science?
}

\section{Untestable ecological theory won't help solve environmental problems.}

\section{Jim} Smith

cientists often dismiss philosophy as a vague, incomprehensible subject bearing little relation to science's 'real world' knowledge. Philosophers, on the other hand, have long debated the basis of scientific knowledge and how 'good' science is practised.

The most significant development in the philosophy of science in the past century was Karl Popper's book Conjectures and Refutations (1963), in which he showed how science can be distinguished from non-science or pseudo-science. Popper argued that all scientific theories are no more than conjectures that are more or less well tested. Any theory may be disproved by observation or experiment and replaced by a new, better theory.

The classic example of the working of good science is relativity theory's overthrow of newtonian mechanics. Relativity was first tested by its prediction that the Sun's gravitational pull would bend starlight, a phenomenon not predicted by Newton. The spectacular agreement between Einstein's predictions and observation of the 1919 solar eclipse were both a refutation of newtonian mechanics and the first test of relativity theory.

Popper emphasized two characteristics of scientific theory. First, a theory makes predictions that can be tested by empirical observation; and second, it is provisional: no matter how often it is tested, it is always possible that a future experiment or observation will contradict it. We cannot claim that relativity theory represents absolute truth because new observations may someday show it to be false. Despite this, we have faith in scientific theory because it can be tested against real data. Nonor pseudo-science, to Popper, is knowledge which is not, or cannot be, tested.

Issues concerning hypothesis testing are particularly relevant for the environmental and ecological sciences, because the complexity of environmental systems often limits our ability to test hypotheses quantitatively. Much 'theoretical' environmental and ecological science is necessarily based on laboratory experiments carried out under very controlled conditions. But little effort goes into extrapolating the results of these studies to the outside world. Theoretical ecologists studying fruitfly populations in the lab would be horrified at the thought of their carefully calibrated models being used to predict the behaviour of wild populations. The assumptions relevant to the laboratory would not hold in a real, complex environment. The models are therefore very weakly

\section{ovelty and innovation are often rewarded at the expense of more useful applied research.}

predictive. Of course there is a place for detailed studies of environmental processes, but such studies will not constitute useful scientific theory until they can be used to make predictions in the real world.

The most pressing environmental problems require scientists to predict the effects of man-made changes. We need, for example, to predict the density and distribution of artificially introduced species; to evaluate the impact on an ecosystem of a roadbuilding programme; or to assess the consequences of pollution. Despite the urgent need for properly tested predictive models, such models are extremely rare. Although the scientific literature teems with mathematical models, these are almost never applied and tested by formal 'blind' predictions of the real-world behaviour of environmental systems.

In his Critique for Ecology (1991), US ecologist Robert Peters argued that environmental and ecological scien- tists have focused on theoretical 'insight' and 'explanation' of complex ecosystems at the expense of predictive solutions to applied problems. Peters believed that "because many of the central issues of ecology are confused or untestable, ecologists are uncertain about what information the science needs". This has resulted in a 'trap of originality' in which novelty and innovation are often rewarded at the expense of applied research which, although it may be less technically brilliant, may produce results more useful to society. Ingenuity and innovation may be characteristics of good science, but they are not a substitute for properly elaborated and tested theory.

Faced with a complex environment, scientists have responded by developing ever more intricate and detailed techniques and models. Yet, ironically, simple semi-empirical relationships are usually a much better guide to the behaviour of environmental systems. Such models have achieved some notable successes: predicting a lake's biological response to phosphorus, for example, was key to justifying, and managing, phosphorus removal from effluents. Similarly, empirically based predictions of habitat requirements guided the successful reintroduction of the large blue butterfly (Maculinea arion) to Britain. Perhaps ecological and environmental scientists should concentrate less on theoretical explanation and more on finding applied solutions to humankind's environmental problems. Jim Smith is at the Centre for Ecology and Hydrology, Winfrith Technology Centre, Dorchester, Dorset DT2 8ZD, UK.

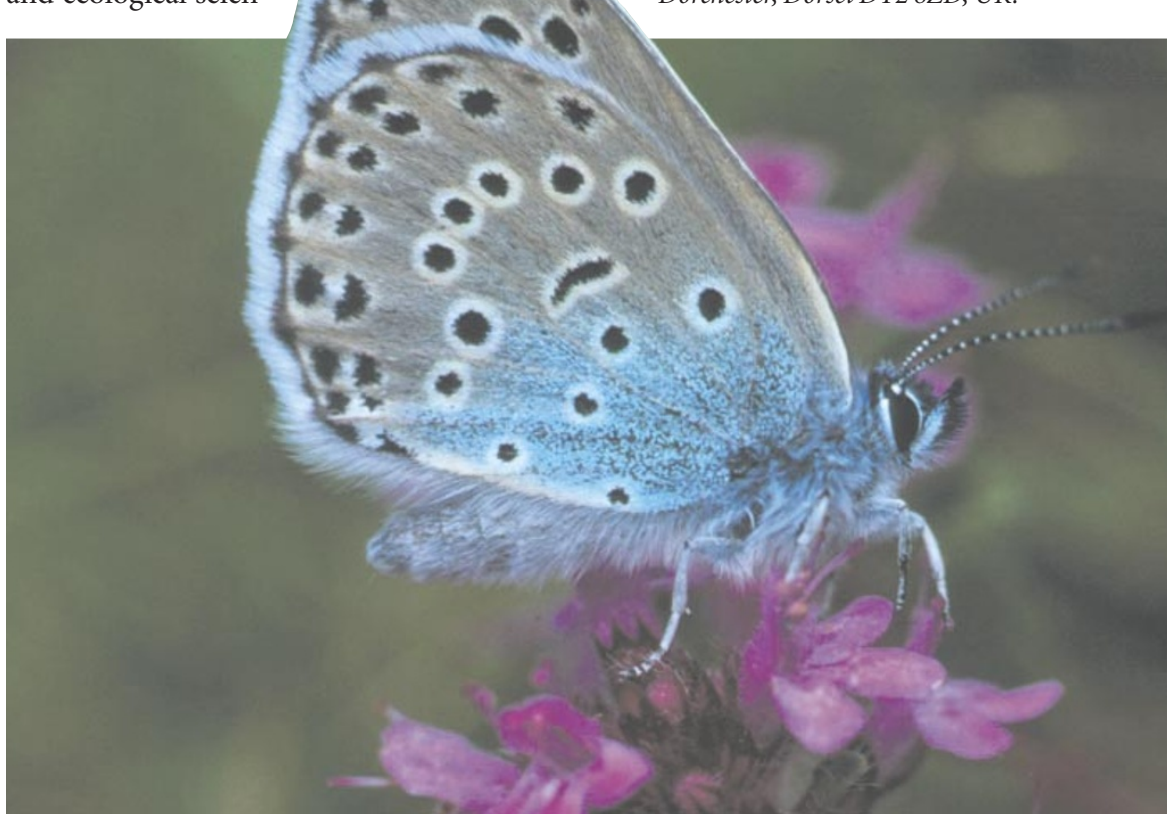

Keep it simple: empirical studies of habitat requirements were the key to saving the large blue butterfly. 